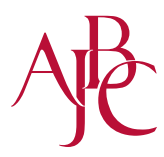

\title{
Application of the Chinese Medicine Theory in Skin Beauty
}

Yu Wang ${ }^{1}$, Wen-Yi Zhu ${ }^{2}$, Hong Meng ${ }^{2 *}$

${ }^{1}$ Beijing University of Chinese Medicine, Beijing, China

${ }^{2}$ China Cosmetic Collaborative Innovation Center, Beijing Technology and Business University, Beijing, China

*Corresponding author: Hong Meng China Cosmetic Collaborative Innovation Center, Beijing Technology and Business University, No. 11/33, Fucheng Road, Haidian District, Beijing, China

Tel. : +86 13691589617

Fax: +86 1068987110

Email: menghong2000@163.com

Received February 23, 2016

Revised May 16, 2016

Accepted May 25, 2016

Published June 30, 2016

\begin{abstract}
Purpose: The Chinese medicine theory is a systematic understanding of the phenomenon of human life and the influential factors. To be aware of the medical system theory in the field of skin beauty in the recent years. Methods: We summarized the application of Chinese medicine, related methods and prescription drugs in skin beauty, and combed the context of application. Result: We elaborated the application from four parts: form, constitution, health maintenance, preventive treatment of disease. Conclusion: Chinese medicine, related methods and prescription drugs were widely used in the field of skin beauty with remarkable results and have guiding significance for the development of skin care products.
\end{abstract}

Keywords: Chinese medicine, Skin, Beauty, Theory, Application

\section{Introduction}

爱美是人的天性, 古人亦如此。殷商记录的“胭脂” , 《礼 记》所载的缄羊脂, 《时后备急方》中的“张贵妃面亳” “白 杨皮散” “令面白如玉色方”，《外台秘要》辟专卷单论美容 的221方, 盛行于宋代的“佛妆” 以及后世大量的养颜技术和 方药, 无一不在说明中医美容史的悠久绵长(Wu, 2015; Gao et al., 1998), 而纵观古代美容的文化传统、历史经验, 其根源都 离不开中医学理论和传统美学观念的指导和实践。

美容皮肤科学是祖国传统医学皮肤美容精华、医学美学、 皮肤科学与美容技术相结合的产物, 是现代皮肤科学中不可 或缺的重要组成部分(Yang et al., 2007)。为了梳理中医学理 论在皮肤美容中的应用脉络, 本文试从形态、体质、养生和 治未病四个方面分别作以论述。

\section{Theoretical background}

\section{Form}

形态科学是生命科学的基础学科。在探索生命本质和生命 现象过程中, 人类不但解释生命现象是什么, 而且不断说明 其为什么(Meng et al., 2003)。一般说来, 具有一定形态结构 的物质才具备某种功能, 没有无物质的功能, 也没有无功 能的物质(Chu \& Li, 2007)。早在《黄帝内经》中, 中医对人 体形态就有论述, 经后世发展, 中医对人体的认知由最初模 糊的实体化, 逐步转向为功能观为主、形态观为辅的理论体 系, 即形态抽象化, 它与中医的生理和病理学浑然一体, 有 效地指导着中医的临床实践。

当今, 中医学和现代医学研究均已表明: 一个完整的生物个 体, 系统之间、器官之间、细胞之间是相互作用和发生影响的 (Mengetal.,2003)。皮肤是人体最大的器官, 位于人体最表面, 除 了保护着我们的全身, 它还是我们的天然外衣, 是构成人体美的 重要标志(He\&Zou, 2013), 其作为美容的主要对象, 亦不例外, 
其形质色泽与构成人体的脏腑、经络、气血津液密切相关。

\section{1) Zang-fu}

脏腑, 是内脏的总称(Yin, 1984)。《素问·五脏别论》明确指 出五脏、六腑、奇恒之腑总称曰“脏腑” (Wang, 2005)。脏腑 的功能是机体生命活动与形体生长的重要基础, 皮肤的生理 与功能依赖于脏腑的功能(Wang \& Xu, 2009)。以下仅论五脏 与皮肤美容的关系。

\section{(1) Heart}

《素问·六节脏象论》曰: “心者, 生之本, 神之变也, 其华 在面, 其充在血脉”《素问·五脏生成篇》曰: “心之合脉也, 其 荣色也” (Wang, 2005)。《灵枢·邪气脏腑病形》提出: “十一经 脉, 三百六十五络, 其血气皆上于面” (Wang, 2004)。由此看 来，心主血脉，其华在面是心与头面皮肤关系的根结所在。 由于面部皮肤的特点是脉络极其丰富, 所以心气心血的盛 衰, 很容易从面部皮肤色泽的荣枯上反映出来(Yu, 2002)。心 的气血充沛, 方能使面色肤色红润光泽。若心血不足, 脉失 充盈, 则面色淡白无华, 甚至枯槁; 心气不足, 血不上荣, 则 面色虚浮㿠白; 血行不畅, 血脉瘀滞, 则面色青紫, 枯槁无 华。心在志为喜。喜是一种积极良好的情感活动, 心情喜悦 往往能面露佳色, 让面部保持红润, 而且有光泽。反之, 如 果情绪不佳, 甚或悲哀、暴怒, 必定花容失色, 使皮肤黯淡 无光 (Yu et al., 2014; Zhang, 2002)。

\section{(2) Liver}

《素问·六节脏象论》曰: “肝者, 罢极之本, 魂之居也, 其华 在爪, 其充在筋。” (Wang, 2005)。肝的主要生理功能是主疏 泄和主藏血, 其生理特性是主升主动, 喜条达而恶抑有(Ling, 2012), 肝对皮肤的影响, 主要通过调节情志和调理血液来实 现的(Zhou, 2008)。

情志活动分属五脏, 虽为心脑元神所统摄, 但离不开肝之疏 泄。只有肝的疏泄功能正常, 人体才能很好的协调自身情志 活动。心情愉悦, 气血和畅, 则全身肌肤濡润光泽, 富有弹 性。同时情志舒畅时, 气血平和, 疏泄通利, 有助于筋目和爪 甲的濡养而神清目明、筋强爪坚。肝在志为怒, 当大怒时, 情绪六奋急燥, 为疏泄太过, 可见面红目赤。肝失疏泄, 情志 不畅, 抑郁不乐时, 又可见皮肤晦暗, 干燥粗䊁, 频发丘疹, 目眶发黄, 色素沉着, 易生黄褐斑, 甚至出现面部皱纹丛生, 筋软甲脆(Zhou, 2008; Xie, 2007)。

血液与人体美容关系密切, “血盛则形盛, 血衰则形萎, 血 败则形坏”。肝藏血, 既可贮藏血液, 又可调节血量, 叶天 士在《临证指南医案》中指出 “女子以肝为先天” , 在美容
方面, 肝的藏血、调血机能对女子尤为重要(Ling, 2012)。肝 藏血量充足则能有效调节血量, 肝促心行血, 使人体血量充 盈, 妇女月经调畅, 面色红润; 若肝藏血不足, 则血虚不能 濡养肌肤、毛发, 可见面色苍白, 皮肤干燥脱屑, 瘙痒, 皲裂 等。肝调血量作用的发挥有赖于的疏泄功能的正常。气为血 之帅, 气行则血行, 若肝失疏泄, 则气的升发不足, 气机的疏 通和发散不利, 血行不畅, 气滞血瘀。出现面色䖿黑, 唇甲青 紫, 皮下紫斑, 肌肤甲错等皮肤异常表现(Zhou, 2008)。

\section{(3) Spleen}

《素问·六节脏象论》曰: “脾……其华在唇四白, 其充在 肌。” (Wang, 2005)。脾位居中焦, 主升清运化, 为气血生化 之源(Gan, 2007), 人体脏腑器官、营卫经络、形体官空、肌 肤皮毛无不仰仗脾胃(Wuetal., 2008)。脾胃功能正常, 则饮食 水谷得以化生精微, 精微得以升散。营养五脏六腑, 五脏精 气充沛则两目畑昫有神, 面色红润光泽; 营养四肢百骸、皮 毛、筋肉, 则筋骨强健, 肌肉满壮, 肤如凝脂(Gan, 2007); 若 脾虚, 则不能将食物转化为精微物质, 人会出现精神萎麼, 面色萎黄, 或面如土色, 晦暗无华(Yu, 2002), 如《中藏经》 云: “脾者肉之本, 脾气已失, 则肉不荣, 肉不荣则肌肤不滑 泽” (Hua, 2011)。也就是说, 脾气健旺, 则脏腑肌肤得到濡 养, 气血得以补益, 肌肤润泽; 反之, 脾失于健运会导致气血 乏源, 脏腑功能低下, 面无光泽, 目无色彩, 毛发枯干, 老象 丛生(Yu, 2002)。脾在志为思。思, 即思考、思虑, 是人体精 神意识思维活动的一种状态。脾志思与心主神明亦有关, 故 《针炎甲乙经》说: “思发于脾而成于心”，正常的思考对机 体的生理活动无不良影响, 但思虑过度、所思不遂会影响气 的正常运动, 形成气结(Shan et al., 2003)。气机郁结则影响 脾气上升, 亦可耗伤心血, 导致心脾两虚。心血不足, 不能荣 养面部皮肤, 则面色苍白无华, 毛发枯楟; 脾失健运, 营养物 质来源不足, 则出现早衰, 面色萎黄无泽, 面部皱纹, 眼睑下 垂, 皮毛憔悴, 脱发等损美之症(Zhang, 2002)。

\section{(4) Lung}

《素问·六节脏象论》曰: “肺者, 气之本, 魄之处也, 其华在 毛, 其充在皮” (Wang, 2005), 指出肺在体合皮, 其华在毛。 皮毛, 包括皮肤、汗腺、毛发等组织, 是一身之表, 也是中医 美容的重要作用对象(He, 2007)。就皮肤而言, 肺的宣肃作用 会加强皮肤的呼吸功能, 促进其吸收（营养成分）、分泌（汗 液及油脂）和排泄（皮肤表层代谢产物）等生理活动的进 行。在肺的功能正常的时候, 尤其是肺的宣发作用, 如《素 问·经脉别论》云: “食气入胃, 浊气归心, 淫精于脉, 脉气 流经, 经气归于肺, 肺朝百脉, 输精于皮毛” (Wang, 2005)。 
肺能将脾所转输的的津液和水谷精微布散到全身, 外达于皮 毛; 另外, 在散气和津液排泄过程中, 还可将皮肤表层代谢 产物排出体外, 从而保证毛空通畅, 肌肤洁净, 焕发自然光 泽。反之, 肺的功能障碍时, 则卫失调控, 导致皮肤汗孔排泄 不畅甚至堵塞, 皮肤新陈代谢产物不能随散气、汗液排出体 外, 使皮肤出现斑点, 或䵢黑或凹凸不平, 甚则面部或胸背部 皮肤产生痤疮(Yu, 2002; He, 2007)。肺在志为悲忧。悲 忧均 属非良性刺激的情绪反应(Shan et al., 2003), 悲忧太甚, 可致 心肺郁结, 意志消沉。肺气虚衰, 不能宣发营卫之气, 津液于 皮毛, 可见面色白无泽, 皮肤枯槁, 皱纹丛生(Zhang, 2002)。

\section{(5) Kidney}

《素问·六节脏象论》日: “肾者主蛰, 封藏之本, 精之处 也, 其华在发, 其充在骨” (Wang, 2005)。肾为先天之本, 主 藏精, 肾所藏的精气是构成和维持人体生长发育及各种生命 活动的重要物质基础, 所以肾精的盈亏与人体强壮及衰老的 关系是密不可分的。肾精对皮肤尤为重要(Yu, 2002), 《素问. 上古天真论》记载了肾中精气充盛与否对肌肤状况的影响:

“女子……五七, 阳明脉衰, 面始焦, 发始邽; 六七, 三阳脉 衰于上, 面皆焦, 发始白……女夫……六八, 阳气衰竭于上, 面焦, 发髸斑白……” (Wang, 2005)。此段中的“面焦”, 即是面部肤质随年龄增长的结果, 肾气是人体先天之气, 其 受后天之气的滋养才能发挥功能, 在人体成长过程中, 体内 的肾气由弱至盛, 再由盛转衰, 使得颜面失养, 而致面容憔 悴, 这正是容颜衰老的客观反映。可见, 容颜衰老的原因之 一就是肾气的衰减, 故驻颜可从补肾着手。肾在志为恐。恐 是一种恐惧、害怕的情志活动。《素问·举痛论》: “恐则气 下, ……惊则气乱。” (Wang, 2005)。惊恐则正气下陷, 导致 上部头面失其营养, 出现面色㿠白, 血色浅淡, 皮肤干燥, 毛发脱落、焦枯或斑秃、变白等症候(Yu, 2002)。

总之, 从脏腑整体的角度看, 五脏六腑的功能正常, 在延 缓衰老, 保持容颜俊美方面起着关键的作用, 若要容貌美, 皮肤美, 必须保养脏腑, 使其功能正常。

\section{2) Meridians}

经络是人体结构的重要组成部分, 具有联络组织器官, 沟 通表里上下, 以通行气血阴阳、感应传导、调节机能活动等 功能的结构系统。经络的这些功能, 具有传递人体中各种信 息的作用, 体内的某种刺激使脏腑功能活动发生变化时, 可 以通过经络的传导而反应于体表皮部, 即所谓 “有诸内, 必 形诸外” (Cheng, 2008)。皮部是经络系统的组成部分, 是 经脉机能活动反映于体表的部位, 也是络脉之气散布之所 在。皮部位于人体最外层, 连接了经络系统的各个组成部
分, 是机体与外界接触的天然屏障(Cai \& Jin, 2012)。皮部 在生理状态下, 具有属脏腑、通经络、固体表、密腠理的作 用, 在病理状态下, 又有传注病邪、反应证候的作用。当外 邪侵袭时, 皮部首当其冲(Wang \& Zhang, 2015), 其受病可 在十二经所通过的部位发生疾病, 或脏腑受病也可反映于相 应的经络, 因而可以根据疾病表现出来的部位, 作为疾病诊 断的依据。皮肤表现出来的红斑、丘疹、水疮、结节、糜烂、 鳞屑等损害, 按照所属的经络循行体表部位来确定某经发 病, 如带状疮疹往往是肝经湿热所致, 故其病损常是肝经行 走的躯干两侧; 若皮损广泛, 分布于数经所过之处, 则以最 初出现的皮损为主(Wang \& Xu，2009)。临床上, 根据皮损所 在皮部、所属经络脏腑之功能进行辨证施治, 对一些皮肤疾 患也有良好的效果(Cai \& Jin, 2012)。可见，经络理论可有效 的指导皮肤美容实践。

\section{3) Qi-blood-body fluid \\ (1) Qi and blood}

气血是维持人体生命活动的基本物质。气，是指体内流动 着的、富有营养的精微物质, 另一是指脏腑功能活动的动 力。其生理功能是熏肤、充身、泽毛。血, 源于先天之精 和饮食物之精华。人体之生理功能、精神意识、无不以血 为基础, 血不足则百脉空虚, 身体衰弱, 百病丛生(Zhang, 1984)。《素问·调经论》曰: “人之所有者, 血与气耳”, 又 云 “血气不和, 百病乃变化” (Wang, 2005), 《圣济总录》 云: “驻颜理容......当以益气血为先” (Zhao, 1962), 分别明 确指出气血对人体及美容的重要性。

皮肤是气血运行与机体内部密切联系的重要器官(Wang \& $X u, 2009)$ 《灵枢·阴阳二十五人》认为人体气血充盈可起 到美容的作用, 曰: “气血盛则髯美”, 并可 “美眉以长、耳色 美” “气血和则美色”。只有气血生成充盈、功能正常, 才能 维持整个人体正常的生理活动, 为人体的健美提供保障(Xie, 2007)。反之, 气血失和, 或偏盛, 或偏衰, 都则可以引起 一系列的病理变化。气血失和于皮肤者多为气虚、气滞、血 虚、血热、血燥、血瘀、气血两亏。气虚可使皮肤不充, 毛发 不泽; 气滞可使气机不畅, 皮肤发生黑斑, 面部出现黄褐斑 等; 血虚可导致肌肤失养, 表现为毛发干枯、肌肤甲错、脱 屑蛮痒、疼痛等; 血热致血液运行加速, 脉管扩张, 或血液 妄行, 皮肤出现灼热潮红、红斑、肿胀、出血、紫斑等; 血燥 可使脉中血液干燥、枯涩, 表现为皮肤瘙痒、干燥、粗糙、 角化、脱屑、皲裂、肥厚、疣状改变等; 气血两亏以致肌肤 失养, 机能衰退, 表现为面色无华、肌肤甲错、或色素沉着 (Wang \& Xu, 2009; Zhang, 1984; Zou, 1995)。 


\section{(2) Body fluid}

津液是津和液的总称, 是由人体脏腑官空所化生的富有 营养的液态物质。津与液有所区别, 津是较清稀、富于营 养的水液, 善于流动, 主要周流于全身; 液是较稠厚, 起濡养、润滑作用的液态物质, 相对稳定, 主要存在于局 部(Yu et al., 2013)。《灵枢·决气》曰: “腠理发泄, 汗出溱 溱, 是谓津……谷入气满, 淖泽注于骨, 骨属屈伸, 泄泽 补益脑髓, 皮肤润泽, 是谓液” “津脱着, 腠理开, 汗 大泄；液脱着，骨属屈伸不利，色天” (Wang, 2004)。皮 肤的润泽紧致需要津液的滋养(Guo \& Zhu, 2015)。正常情 况下，津液渗透于皮肤腠理之间，以滋润营养皮肤，使 皮肤潮润, 肌肉丰满, 毛发光润, 口唇润泽(Zou, 1995); 反之津液亏虚则皮肤失于濡养引起干燥, 皮肤现干枯、 干癔、皱纹、脱屑、皲裂、少泽等(Wang \& Xu, 2009); 津液 输布、排泄障碍则致湿浊中阻、痰饮凝聚、水液渚留(Sun, 2007), 皮肤表现为结节、肿胀、水疮等(Zhang, 1984)。 从以上可看出, 皮肤的生理活动依赖于气血津液的功能, 皮肤的生长及代谢有赖于气血津液的功能。气血津液的虚实 变化、各自的代谢或运动失常均与皮肤病的发生及发展有着 密切的关系(Wang \& Xu, 2009), 皮肤美容当可从此辨证论治。

\section{Constitution}

体质是人的生命活动的重要表现形式, 是个体在先天禀赋 和后天获得的基础上所形成的形态结构、生理功能和心理状 态方面综合的、相对稳定的固有特质, 是人类在生长发育过 程中所形成的与自然、社会环境相适应的人体个性特征(Liao et al., 2008)。诚如“世界上没有两片完全相同的树叶” 样, 不同的个体也有不同的体质。体质的差异, 决定着体 型、容貌、气质的不同，而肤质的优劣又是容貌最直接的反 映, 它的形成与先天遗传与后天获得密切相关。

\section{1) Natural endowment}

禀赋的概念, 《辞海》: “禀赋, 犹天赋, 指人所禀受的天资 或体质”。《现代汉语词典》释禀赋为: “人的体嵬、智力等方 面的素质”。《中医大辞典》则把 “禀赋” 解释为 “先天赋予 的体质因素” (Wang \& Liu, 2006)。中医禀赋理论的起源可以追 溯到《灵枢·天年》中：“人之始生，……目为基，以父为 循” (Liu et al., 2011), “血气已和, 荣卫已通, 五脏已成, 神气舍心，魂魄毕具，乃为成人” (Wang, 2004)。每个人的 容貌与体型与生俱来都带有父母遗传的特征。身长之高矮、 五官之形状、皮肤之颜色等个体外在形象皆主要取决于先天 禀赋。大抵胎儿或幼儿时候的皮肤色泽形质都多与母体密切 相关, 母体的内外环境因素对于胎儿先天禀赋的形成具有重 要影响。一般情况下, 孕妇健康则胎儿健康, 皮肤亦无碍。
孕妇身体虚弱或身心有疾则会向胎儿传递不良信息, 甚至导 致各种疾病的产生(Wang \& Liu, 2006)。如《幼幼集成》云:

“如禀肺气为皮毛, 肺气不足, 则皮薄怯寒, 毛发不生; 禀 心气为血脉, 心气不足, 则血不华色, 面无光彩; 禀脾气 为肉, 脾气不足, 则肌肉不生, 手足如削……此皆胎禀之 病” (Yang, 2006)。所以, 身心健康的母亲、和谐的生态环境 是形成子代优良体质必不可少的组成部分(Liu etal., 2007)。

\section{2) Acquired}

肤质的优劣是机体内外环境等多种复杂因素共同作用的结 果, 既受先天因素制约, 又受后天因素的影响。后天因素包括 自然环境、社会环境及生活方式, 这些因素对肤质具有重要影 响。如皮肤的紧致松弛、肤色的明暗深浅、皮肤纹理的粗细无 不与运动之多寡、奉养之优劣、教育之多少密切相关。在自然 环境方面, 人类生存的自然环境逐步恶化是不争的事实, 生态 平衡受到破坏，大量的污染物通过皮肤、口鼻侵入机体，产生 机体损伤, 气血阻滞, 容颜失泽, 表现为皮肤油淢不洁、肤色 暗黄、毛孔粗大、皮肤粗粗、痊疮等。在社会环境方面, 生存 竞争带来的心理压力, 其中压抑、郁怒、忧愁、思虑容易造成 气机上下郁滞, 累及皮肤则表现为座疮, 黄褐斑, 皮肤油㖑粗 䊁等。在生活方式方面，饮食结构的变化也会影响到皮肤的状 态。近年来, 中国人的饮食结构发生了巨大的变化, 主要表 现在过食肥甘厚淢, 恣食辛热香浓, 多食冰镇冷饮。《素问. 奇病论》曰: “肥者令人内热, 甘者令人中满。”长期肥甘厚 淢、恣食辛热香浓易形成湿热、痰湿体质, 皮肤表现为面垢油 光、黄褐斑、易生痤疮粉刺等。多食冰镇冷饮，对于机体的影 响一是伤及脾胃阳, 二是引起血行不畅, 而趋向痰湿、瘀血体 质, 皮肤表现为肤色较暗, 多油淢, 黄褐斑, 面部或有雀斑, 黑眼圈等(Fu, 2002)。此外, 饮食的嗜好也会影响皮肤, 如《素 问·五脏生成篇》曰: “多食咸, 则脉凝泣而变色; 多食苦, 则 皮槁而毛拔; 多食辛, 则筋急而爪枯; 多食酸, 则肉胝皱而唇 揭; 多食甘，则骨痛而发落” (Wang, 2005)。

\section{Health maintenance}

养生又称摄生、道生, 最早见于《庄子》，是通过养精神、 调饮食、练形体、慎房事、适寒温等各种方法去实现的一种综 合性的强身益寿活动(Cen, 2001)。《庄子.内篇》记载, 所谓 “养” ，即保养、调养、补养、护养之意; 所谓 “生” ，即 生命、生存、生长之意。“养生”的内涵，一是如何延长生 命的时限, 二是如何提高生活的质量(Dong et al., 2009)。中 医养生相对于其他养生而言, 其发生发展受到我国古代哲学 思想的影响和中医基本理论的指导。从功能上讲, 养生可以 说是一种作用于人体的整体干预, 它要求人们顺应自然四时 
的变化, 调和形体与精神的关系, 沟通人体各部的联系, 使 人体处于天人相应、内外一致的最佳状态(Tan \& Liu, 2008)。 当代美学家蔡仪曾说过: “在自然美中, 人体美的完整性最 强, 人体的各个部分都是互相紧密联系的，而且每一个部分 都完全从属于整个个体”(Cai, 1985)，这正强调了整体美的重 要性, 而皮肤美容的目的不单是局部的修饰, 更重要的是局 部与整体的协调, 即整体效应的提升。可见, 从此视角看, 皮肤美容和中医养生是契合的, 并且主要体现在两方面。

\section{1) Syncretism between heaven and man}

天人合一思想可以溯源到商周时代，《礼记·表记》云: “殷人尊神，率民以事神”，此时的天人关系即神人关系 (Zhao \& Zhao, 2010)。此后，《管子·五行》又提出 “人与 天调”。到宋代，张载明确提出 “天人合一”的命题。这 个命题强调人与自然的和谐统一、渗透交融(Tan \& Liu, 2008; Huang \& Yan, 2000)。“天人合一”哲学思想对中医学和中国 传统美学都有重大影响。中医学认为人是自然的一部分, 自 然环境的变化对人体有影响, 反之, 人的行为若违背自然规 律, 也不能维持自身的健康(Huang \& Yan, 2000)。纵观 “春夏 养阳，秋冬养阴” “顺时起居” 等在 “天人合一”指导下建 立起来的顺应自然的养生原则与方法, 其在追求养生的过程 中，也不自觉地热衷美容，譬如使人 “面如童子” “色如少 女” “发白再黑” 等论述在古代中医养生书籍中比比皆是, 这正体现了自然养生与自然美容的统一。

2) Holism of body and spirit

形和神是标志人的结构和生命本质的一对范畴。在该观点的 影响下，中医养生在形神一体的整体思维模式下，建构了更 为客观和全面的形神共养的养生原则和方法, 主要有 “形神 共养” “保养真气” “调畅情志” 等(Tan \& Liu, 2008)。中医美 容也追求形神一体，即形体美与神韵美的统一，当一个人具 有在健康基础上的美的形体的同时，又具有潇酒的气质和高 尚的品德时，会被认为是最理想的美。神形美不仅是人的躯 体和心理健康的表现，而且美的形体通过人的感官作用于人 体，使人愉悦，美的精神通过人的情志作用于人体，使人积 极向上, 又对人的健康起推动作用(Huang \& Yan, 2000)。

\section{Preventive treatment of disease}

“治未病”一词首见于《素问·四气调神大论》: “是故圣 人不治已病治未病，不治已乱治未乱，此之谓也” (Wang, 2005)。“治未病”有狭义和广义之分。狭义是指治疗疾病的 早期状态。广义主要包括治其未生, 防患未然; 治其未成, 防微杜渐; 治其未传, 防护于先; 治其未发, 把握时机; 㽿
后调摄, 防其复发; 用药精当, 勿使过用(Xu \& Guo, 2008)。 究其内涵, 概而言之, 就是指在不同情况下, 均应预先采取 一定措施防止疾病的发生与发展(Shang, 2008)。皮肤功能的 正常与否直接反映出着皮肤形质色泽的程度, 其异常状态 属于中医 “治未病” 中的“未病”阶段。皮肤美容是皮肤功 能维护与修复之于 “治未病” 思想的现实体现, 在具体实施 “治未病” 思想时, 主要在于未病先防, 即 (1)鉴别和认识皮 肤健康的基本表象或早期变化。皮肤外观活力主要表现在光 泽、形态、质感等皮肤信息，中国人正常的皮肤表征是 “红 黄隐隐, 明润含蓄”, 这是皮肤生理功能正常的综合体现, 亦是皮肤功能与美感异常的重要早期鉴别征象, 以及脏腑、 经络、气血津液功能活动正常的体表征象; (2)预防有损皮肤 健康美感的机体内外因素。皮肤位于一身之表, 时刻经受和 反映着内外在环境的变化, 其色泽形质是体内外因素共同作 用的结果, 对比 “红活荣润” 观察不同皮损状况对早期防范 干预具有重要意义(Lu, 2008)。

\section{The application of different skin care methods in Chinese medicine theory}

\section{Skin beauty and meridian treatment}

经络护肤是指在人体经络所流布循行的穴道上, 使用 指压术或针刺或者多、燔、烤、刮等刺激的美容法。经 络是运行全身气血、联络脏腑肢节、沟通上下内外的通 路，人体营卫出入、气血流通、津液运化、气机升降等 都是通过经络来实现的。经络既是联系身体各组织的 通道, 又运行营养物质于全身, 所以经络发生病变就 会影响各器官功能, 各器官发生的病变又可通过经络 反映出来。通过刺激经络, 疏通经络气机, 就可以达到 治疗疾病和美容的目的，如社除色斑、痤疮、皱纹等。

经络美容不仅指脸部的美容而已，还包括全身的健康。其 主要优势在于非药物性调理, 无副作用, 有见效快及标本兼 治的效果。当面部出现黄褐斑时, 原因不仅是皮肤上的问 题, 也与脏器功能失调有关。通过整体调节令经络畅通, 阴 阳协调, 提高和增强机体功能, 可有效地解决美容问题。

\section{Skin beauty and qi and blood}

气血理论指导皮肤养护, 有着坚实深刻的理论基础。人体 血气密不可分。气为阳, 血为阴, 白天阳气生发, 气推动血 行, 若辅以具活血功效的皮肤保养品, 使气血更顺畅地周流 全身，增强皮肤血液循环，输送更多营养物质到面部，同 时帮助推出面部积累的毒素, 使面部皮肤健康红润, 事半功 
倍; 夜晚阳气收玫, 此时宜养血之元, 气之母, 修复白天由 日晒、污染、自由基等给皮肤造成的损害。如此循环往复, 使皮肤达到滋养的良性循环, 呈现健康光泽和肤质。这就是 “日间活血, 夜间养血” 的护肤理念, 是中医传统理念在皮 肤养护方面的延伸。

\section{Skin beauty and constitution}

中医体质类型划分的依据是, 用中医病理表现特点来确定病 理体质分型, 因为体质理论本身是中医认知论证的特色, 中医 九中体质包括平和质、气虚质、阴虚质、阳虚质、湿热质、气 郁质、痰湿质、血瘀质、特禀质。平和型以正常预防为主, 注 意美白、防晒和保湿; 气虚型常无力、阳虚型最怕冷注意防衰 老; 阴虚型最怕热注意保湿补水; 湿热型爱出油、痰湿型易肥 胖, 皮肤油淢易生痤疮; 气郁型爱失眠; 血瘀型易健忘两者皮 肤都容易暗沉、色斑发生; 特禀型会过敏等。

\section{Conclusion}

纵观古代美容的文化传统、历史经验, 其根源都离不开中 医学理论和传统美学观念的指导和实践。本文从形态、体 质、养生和治未病四个方面充分论述了中医学理论在皮肤美 容中的应用, 这对于指导护肤品的开发具有实际指导意义。 融会中医药理论于护肤品中是开发中国特色创新型化妆品的 源泉所在。再者, 秉承中国的传统文化以及博大精深的中医 药传统理论, 推陈出新, 赋予新的开发理念与技术, 可全面 提升产品品质内涵。

\section{References}

Cai Y. Aesthetic principle. Hunan People's Publishing House, Changsha, p81, 1985.

Cen ZB. The cultural connotation of traditional Chinese medicine. Lingnan Culture and History, 2001: 13-22, 2001.

Chu GX, Li QZ. On traditional Chinese medical morphology. Acta Universitatis Traditionis Medicalis Sinensis Pharmacologiaeque Shanghai, 21: 28-32, 2007.

Cai YG, Jin L. The present study on skin diseases by the theory of twelve cutaneous regions. Journal of Practical Dermatology, 5: 282-284, 2012.

Cheng SR. Analysis of clinical application on the theory of twelve meridional dermomeres. Journal of Nanjing
Institute of Physical Education: Natural Science, 7: 13-15, 2008.

Dong YM, Meng H, He CF. Application of Chinese medicine theory and technology in cosmetics. Detergent \& Cosmetics, 32: 14-18, 2009.

$\mathrm{Fu} J \mathrm{Y}$. Human constitution and cosmetology. Chinese Journal of Aesthetic Medicine, 11: 527-529, 2002.

Gao DH, Li JG, Shang KP. The history of aesthetics in traditional Chinese medicine. Chinese Journal of Aesthetic Medicine, 7: 16-18, 1998.

Gan NF. Therapy on liver and spleen for beauty damaging diseases. Lishizhen Medicine and Materia Medica Research, 18: 1219-1220, 2007.

Guo YY, Zhu JT. A preliminary discussion of theory on treatment of xerosis cutis from damp evil. Journal of Chengdu University of Traditional Chinese Medicine, 38: 111-113, 2015.

He L, Zou X. New concept of cosmetic dermatology. Practical Journal of Clinical Medicine, 10: 43-44, 2013.

$\mathrm{He}$ Y. Explore the application and meaning of traditional Chinese medical beauty treatment on lung. Chinese Journal of Aesthetic Medicine, 16: 548-550, 2007.

Hua T. Zhongzang jing. China Medical Science and Technology Press, Beijing, p37, 2011.

Huang FL, Yan ZA. Beauty of human body in traditional Chinese medicine cosmetology. China Journal of Traditional Chinese Medicine and Pharmacy, 15: 8-11, 2000.

Lu Q. The application of the "prevention diseases" of traditional Chinese medicine's concept to cosmetic dermatology. Chinese Journal of Aesthetic Medicine, 17: 1515-1517, 2008.

Ling TH. Brief discussion about liver and traditional Chinese medicine beautification. Journal of Liaoning University of Traditional Chinese Medicine, 14: 8991, 2012.

Liu XZ, Wang XZ, Wang YY. Relationship between innateness and constitution. Journal of Beijing University of Traditional Chinese Medicine, 34: 441-443, 2011.

Liu XZ, Guo L, Wang XZ, Wang YY. Discussion on innate essence and representation after birth of innateness. Journal of Beijing University of Traditional Chinese Medicine, 30: 587-589, 2007.

Liao Y, Wang Q, Zheng D. Discussion on correlation 
between constitution and beauty-damaging diseases. Shandong Journal of Traditional Chinese Medicine, 27: 149-150, 2008.

Meng YC, Li PT, Bi CE. Study of the relationship between TCM and morphology. Journal of Beijing University of Traditional Chinese Medicine, 26: 8-9, 2003.

Shan DH, Zheng XN, Wang DS. Relationship between traditional Chinese medical five minds, five organs, five body theory and beauty. Liaoning Journal of Traditional Chinese Medicine, 30: 75, 2003.

Sun GR. Basic theory of traditional Chinese medicine. Chinese Medicine Publishing House of China, Beijing, p270, 2007.

Shang QX. Discussion on theory of preventive treatment of disease from traditional Chinese medicine. Shandong Journal of Traditional Chinese Medicine, 27: 226-228, 2008.

Tan YY, Liu ZC. The construction of traditional Chinese medicine theoretical system. Journal of Shandong University of Traditional Chinese Medicine, 32: 4548, 2008.

Wu YJ. Review on origin and development of traditional Chinese medicine cosmetology in ancient China. Journal of Zhejiang University of Traditional Chinese Medicine, 39: 238-242, 2015.

Wang HM, Xu LM. On traditional Chinese medical physiology and pathology of the skin. Chinese Journal of Dermatovenerology of Integrated Traditional and Western Medicine, 8: 56-58, 2009.

Wang HT. Huangdi neijing suwen bai hua jie. People's Medical Publishing House, Beijing, pp3-326, 2005.

Wang HT. Huangdi neijing lingshu bai hua jie. People's Medical Publishing House, Beijing, pp40-381, 2004.

Wang XF, Zhang XY. The disquisition on cutaneous region theory of traditional Chinese medicine. Journal of Traditional Chinese Medicine, 56: 821-824, 2015.

Wang YY, Liu XZ. The understanding and explanation about the concept of born gift. Zhejiang Journal of Traditional Chinese Medicine, 41: 561-563, 2006.

Wu JD, Ren QL, Gu W, Li SH. The relationship between deficiency of qi and skin aging. Chinese Journal of Aesthetic Medicine, 17: 1371-1373, 2008.

Xie L. Brief discussion on "liver controlling conveyance and dispersion" and traditional Chinese medical beauty. Journal of Liaoning University of Traditional Chinese Medicine, 9: 51-52, 2007.

$\mathrm{Xu}$ YS, Guo YM. Comprehend and reflect the thinking of "preventive treatment of diseases" on huangdi neijingdiscuss the guidance for prevention and treatment of sub-health. Chinese Archives of Traditional Chinese Medicine, 26: 1807-1809, 2008.

Yang S, Wang HL, Zhang XJ. Cosmetic dermatologypast, present and prospects. Chinese Journal of Aesthetic Medicine, 16: 581-584, 2007.

Yin HH. Basic theory of traditional Chinese medicine. Shanghai Science and Technique Publishing House, Shanghai, p28, 1984.

$\mathrm{Yu}$ L. Mechanisms between five internal organs and skin aging. Chinese Archives of Traditional Chinese Medicine, 20: 779-780, 2002.

Yu RX, Han L, Qin LL, Wu LL, Sun W, Liu TH. Analysis of Chinese medicine theory and methods of protecting and beautifying skin. Journal of Liaoning University of Traditional Chinese Medicine, 16: 104-107, 2014.

Yu HL, Zheng Y, Ju HY, Sang XS. Analysis on body fluid theory of Chinese medicine. Information on Traditional Chinese Medicine, 30: 3-5, 2013.

Yang JP. Youyou jicheng. People's Medical Publishing House, Beijing, p86, 2006.

Zhang YX. The seven emotions and cosmetology. Liaoning Journal of Traditional Chinese Medicine, 29: 240241, 2002.

Zhou D. Female beauty focus on liver. Shanxi Journal of Traditional Chinese Medicine, 24: 60-61, 2008.

Zhang ZL. Clinical application of qi-blood zheng differentiation in dermatology. Journal of Traditional Chinese Medicine, 3: 47-51, 1984.

Zhao J. Shengji zonglu. People's Medical Publishing House, Beijing, p3072, 1962.

Zou ZD. Discussion on the theoretical basis of beauty in "neijing (the internal canon of medicine)". Beijing Journal of Traditional Chinese Medicine, 6: 42-44, 1995.

Zhao Y, Zhao JP. Holism of human being and universe on TCM. China Journal of Traditional Chinese Medicine and Pharmacy, 25: 1407-1410, 2010. 


\section{국문초록}

\section{피부미용 분야에서 중의학이론의 응용}

왕유 ${ }^{1}$, 주문이 ${ }^{2}$, 맹홍 ${ }^{2 *}$

${ }^{1}$ 북경중의학대학, 북경, 중국

${ }^{2}$ 북경공상대학 중국화장품공동혁신센터, 북경, 중국

목적: 중의학이론은 중의학이 인간 삶의 현상과 이에 영향력을 미치는 요인들을 체계적으로 이해하는 이론이다. 그러므로 본 연구는 중의학이론을 피부미용으로의 응용에 대해 체계적으로 논술하기 위함이다.

방법: 중의학이론의 기초하에 중의학 연관방법과 중의학 처방이 피부미용에의 응용에 대해 체계적으로 논술하였다. 그리고 중의학 이론의 피부미용에서의 응용맥락을 간단하게 논술하였다.

결과: 본 연구는 형태학, 양생, 체질학, 질병의 예방치료 등 방면으로 중의학이론의 피부미용에서의 응용을 체계적으로 논술하였다. 결론: 중의학이론, 관련방법, 처방은 피부미용분야에 광범위하게 응용되었고 또한 현저한 성과를 거두었으며 앞으로의 피부 케어 제품의 개발에 중요한 지도적 의의를 가질 것이라고 사료된다.

핵심어: 중의학, 피부, 미용, 이론, 운용 


\section{中文摘要}

\section{中医理论在皮肤美容中的应用}

王瑜 ${ }^{1}$, 朱文驿 ${ }^{2}$, 孟宏 ${ }^{2 *}$

1 北京中医药大学, 北京, 中国

${ }^{2}$ 北京工商大学 中国化妆品协同创新中心，北京，中国

目的: 中医学理论是中医学对人体生命现象和相关影响因素的系统认识。系统认识中医学理论在皮肤美容领域的应用。

方法: 用中医学理论的指导, 总结中医学相关方法、方药在皮肤美容领域的应用。对中医学理论在皮肤美容中的应用脉络进行 了梳理并简要论述。

结果: 从形态、体质、养生和治未病四个方面论述了中医学理论在皮肤美容中的应用。

结论: 中医学理论在皮肤美容领域广泛使用，成果显著，对于护肤品的开发具有指导意义。

关键词: 中医学，皮肤，美容，理论，运用 